\title{
Combined optical and mechanical scanning in optical-resolution photoacoustic microscopy
}

Lei Li, Chenghung Yeh, Song Hu, Lidai Wang, Brian T. Soetikno, et al.

Lei Li, Chenghung Yeh, Song Hu, Lidai Wang, Brian T. Soetikno, Ruimin Chen, Qifa Zhou, K. Kirk Shung, Konstantin I. Maslov, Lihong V. Wang, "Combined optical and mechanical scanning in optical-resolution photoacoustic microscopy," Proc. SPIE 8943, Photons Plus Ultrasound: Imaging and Sensing 2014, 89435X (3 March 2014); doi: 10.1117/12.2038693

SPIE. Event: SPIE BiOS, 2014, San Francisco, California, United States 


\title{
Combined optical and mechanical scanning in optical-resolution photoacoustic microscopy
}

\author{
Lei $\mathrm{Li}^{\mathrm{a}}{ }^{\mathrm{a}}$, Chenghung Yeh ${ }^{\mathrm{a}, \mathrm{b}}$, Song $\mathrm{Hu}^{\mathrm{b}}$, Lidai Wang ${ }^{\mathrm{b}}$, Brian T. Soetikno ${ }^{\mathrm{b}}$, Ruimin Chen ${ }^{\mathrm{c}}$, Qifa Zhou, \\ K. Kirk Shung ${ }^{\mathrm{c}}$, Konstantin I. Maslov ${ }^{\mathrm{b}}$ and Lihong V. Wang ${ }^{\mathrm{a}, \mathrm{b}^{*}}$ \\ ${ }^{a}$ Department of Electrical and System Engineering, Washington University in St. Louis, One \\ Brookings Dr., St. Louis, MO, 63130; \\ ${ }^{\mathrm{b}}$ Optical Imaging Laboratory, Department of Biomedical Engineering, Washington University in St. \\ Louis, One Brookings Dr., St. Louis, MO, 63130 \\ ${ }^{c}$ Resource Center for Medical Ultrasonic Transducer Technology, Department of Biomedical \\ Engineering, University of Southern California, 1042 Downey Way, DRB 136, Los Angeles, \\ California 90089-1111, USA \\ *Corresponding author: lhwang@wustl.edu
}

\begin{abstract}
Combined optical and mechanical scanning (COMS) in optical-resolution photoacoustic microscopy (OR-PAM) has provided five scanning modes with fast imaging speed and wide field of view (FOV). With two-dimensional (2D) galvanometer-based optical scanning, we have achieved a $2 \mathrm{KHz}$ B-scan rate and $50 \mathrm{~Hz}$ volumetric-scan rate, which enables real-time tracking of cell activities in vivo. With optical-mechanical hybrid 2D scanning, we are able to image a wide FOV $\left(10 \times 8 \mathrm{~mm}^{2}\right)$ within 150 seconds, which is 20 times faster than the conventional mechanical scan in our second-generation OR-PAM. With three-dimensional mechanical-based contour scanning, we can maintain the optimal signal-to-noise ratio and spatial resolution of OR-PAM while imaging objects with uneven surfaces, which is ideal for fast and quantitative studies of tumors and the brain.
\end{abstract}

Keywords: Medical and biological imaging, Photoacoustic imaging, Scanning microscopy, Three-dimensional microscopy.

\section{INTRODUCTION}

Optical-resolution photoacoustic microscopy (OR-PAM), based on the photoacoustic effect [1], detects optical absorption contrasts with greater sensitivity than all other optical imaging modalities [2]. The high detection efficiency of intrinsic optical absorbers (e.g., oxy-hemoglobin ( $\mathrm{HbO} 2)$ and deoxy-hemoglobin (HbR) $[3,4]$ ) enables OR-PAM to provide label-free high-resolution [5,6] functional imaging of fine structures [7]. OR-PAM has already demonstrated broad biomedical applications in the fields of neurology [8], ophthalmology [9-12], and vascular biology [13]. However, the imaging speed of second-generation (G2) OR-PAM is insufficient for tracking certain fast dynamic activities in vivo [14]. Here, we report a combination of optical and mechanical scanning OR-PAM (COMS-OR-PAM), which provides five scanning modes with fast imaging speed and a wide field of view (FOV).

\section{METHOD}

The COMS-OR-PAM system (Fig. 1) employs a wavelength-tunable laser system (INNOSLAB, Edgewave BX2II-E) for photoacoustic irradiation. The output laser beam is first attenuated by a variable neutral density filter, reshaped through an iris, and then focused by a lens. The focused beam passes through a 50 - $\mu \mathrm{m}$-diameter pinhole for spatial filtering. The filtered beam is launched into a single-mode optical fiber coupler. The fiber output is collimated by a doublet. The collimated beam, reflected by a two-dimensional (2D) galvanometer, fills the back aperture of a doublet (AC127-025-A, Thorlabs). A compensation lens (LA1207-A, Thorlabs) is placed beneath the imaging doublet to achieve nearly diffraction-limited optical focusing in water. A beam sampler and a fast photodiode are inserted between the lens and pinhole to monitor the fluctuation in laser intensity. COMS-OR-PAM employs a ring-shaped focused ultrasonic transducer for reflection-mode ultrasound detection. The ring transducer (35 MHz center frequency, $25 \mathrm{MHz}$ bandwidth, from the Resource Center for Medical Ultrasonic Transducer Technology at the University of Southern California) has a

Photons Plus Ultrasound: Imaging and Sensing 2014, edited by Alexander A. Oraevsky, Lihong V. Wang, Proc. of SPIE Vol. 8943, 89435X - (C) 2014 SPIE · CCC code: 1605-7422/14/\$18 · doi: 10.1117/12.2038693 
2-mm-diameter hole in the center for the optical illumination beam to pass through and provides an acoustic focal diameter of $\sim 90 \mu \mathrm{m}$. The optical and acoustic foci are aligned to maximize the detection sensitivity, and the transducer is submerged in water contained in a plastic tank for acoustic coupling. An imaging window at the bottom of the water tank is sealed with a polyethylene membrane for optical and acoustic transmission.

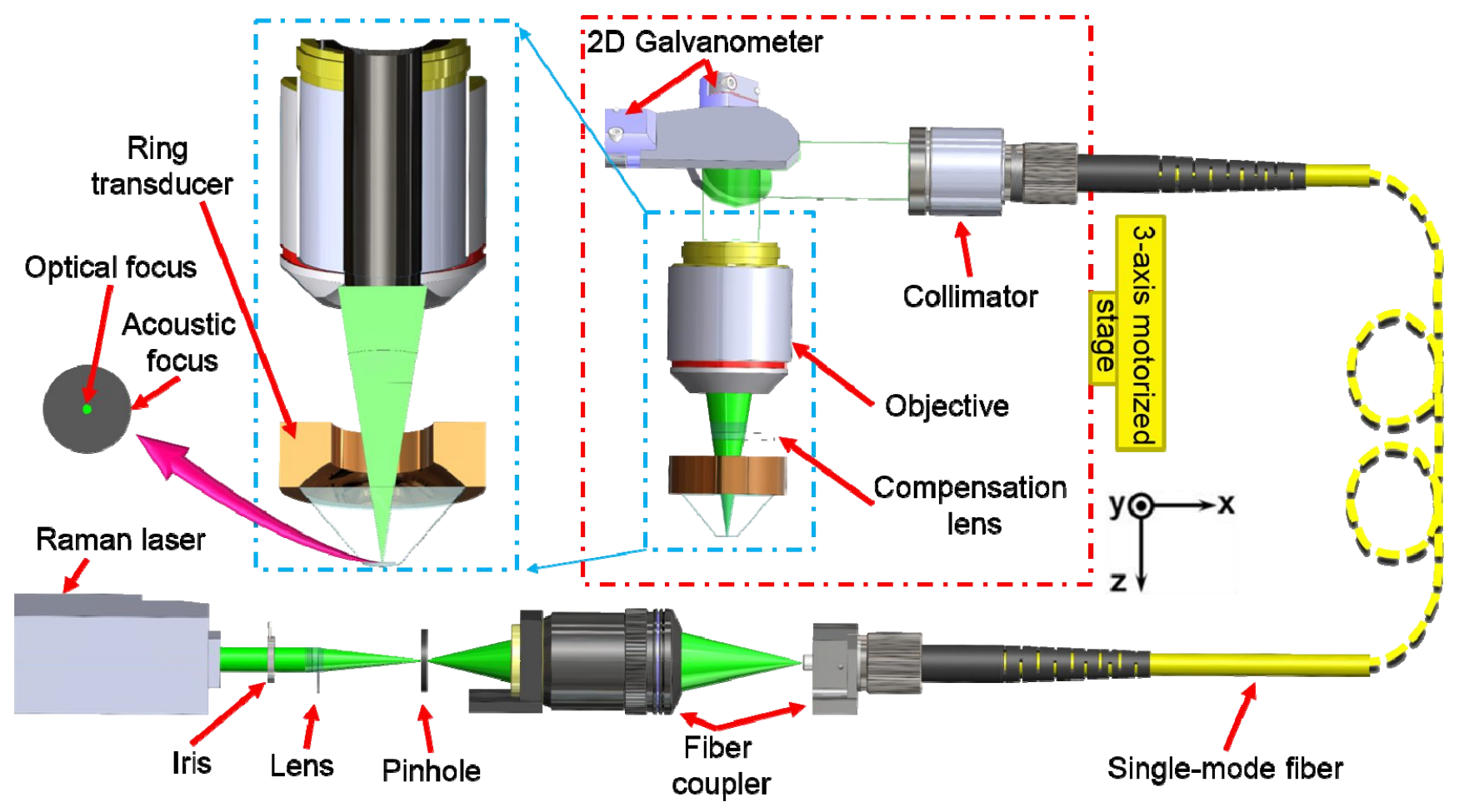

Figure 1. Schematic of the combination of optical and mechanical scanning optical resolution photoacoustic microscopy (COMS-OR-PAM).

\section{RESULTS AND CONCULSION}

COMS-OR-PAM's lateral resolution (quantified in full width at half maximum), determined by the numerical aperture (NA) of the water-immersed imaging doublet $(\mathrm{NA}=0.13)$, was calculated to be $2.1 \mu \mathrm{m}$ at $532 \mathrm{~nm}$. It was experimentally quantified to be $2.6 \mu \mathrm{m}$ (Fig. 2), which is slightly worse than the theoretical value due to the imperfect compensation of aberration.

The whole scanning head is mounted on a 3-axis motorized translation stage ( $\mathrm{X}$ and $\mathrm{Y}$ axes, PLS-85, PI miCos GmbH; $\mathrm{Z}$ axis, KR15, THK. CO., LTD). The three-dimensional (3D) translation stage for mechanical scanning, in combination with the 2D galvanometer for optical scanning, provides five scanning modes: (1) 1D motion-mode (M-mode) mechanical scanning, (2) 2D mechanical scanning, (3) 3D mechanical contour scanning, (4) 2D optical scanning, and (5) synchronized 1D optical and 2D mechanical hybrid scanning.

One-dimensional M-mode mechanical scanning and 2D mechanical scanning are inherited from previously realized in second generation OR-PAM [14] as the basic functions of OR-PAM.

To get optimal sensitivity and resolution of OR-PAM, the features of interest should be in the optical and acoustic confocal region (generally $30 \mu \mathrm{m}$ in length in the axial direction). However, due to uneven tissue surfaces (e.g., tumors or brain), mode 2 scanning cannot capture all the features of interest within the confocal region.

Here, 3D mechanical contour scanning (raster scanning with axial adjustment [15], mode 3) was integrated in COMSOR-PAM to overcome this limitation by acquiring optimally focused 3D images. The sample was first scanned in mode 2 with a coarse step size $(2.5 \mu \mathrm{m} \mathrm{X}$-axis motor step size, $50 \mu \mathrm{m}$ Y-axis motor step size). The maximum-amplitude position in each A-line was identified for each B-scan, and then a polynomial function was employed to fit the set of 
maxima for the 2D curved surface into a coarse map. The coarse map is further linearly interpolated along the $\mathrm{Y}$ direction to reduce the interval to $5 \mu \mathrm{m}$ and keeps the same $\mathrm{X}$-axis step size $(2.5 \mu \mathrm{m})$. Last, the 3 -axis motorized translation stage scans according to the refined contour map. A tumorous mouse ear with uneven surfaces [Fig. 3(a)] was imaged in vivo in modes 2 and 3 to prove the capability of contour scanning.

The tumor region does not show up in the image acquired in mode 2 [Fig. 3(b)] due to the out-of-focus effect. Comparatively, the image acquired in mode 3 [Fig. 3(c)] reveals the vasculature of both the mouse ear and the tumor with resolved capillaries. We experimentally demonstrated that the mode 3 scanning can always keep the features of interest in the optical and acoustic confocal region with maintained signal-to-noise ratio (SNR).
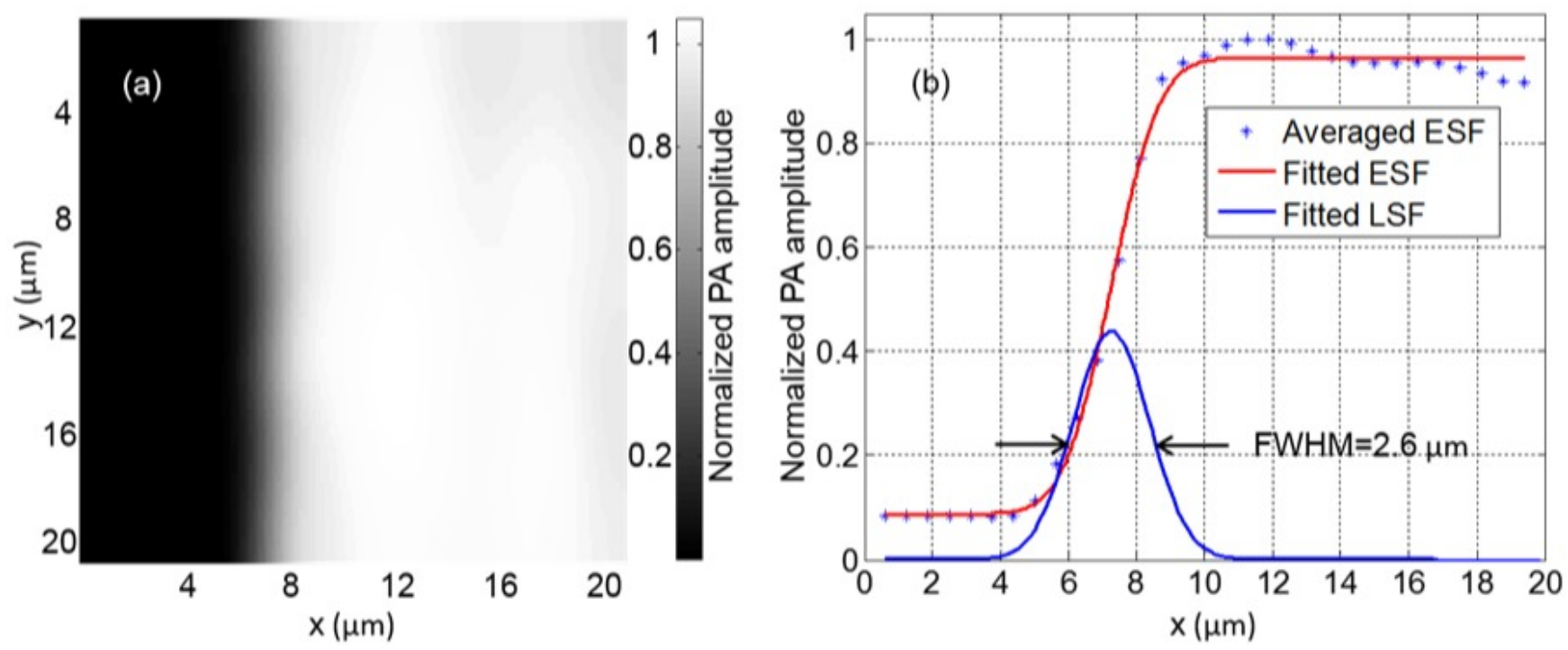

Figure 2. Quantification of the lateral resolution of the CMOS-OR-PAM system. (a) Maximum-amplitude-projection (MAP) image of a sharp-edged metal square acquired with CMOS-OR-PAM. (b) Edge spread function (ESF) extracted from (a) and line spread function (LSF) obtained by taking the derivative of the ESF.
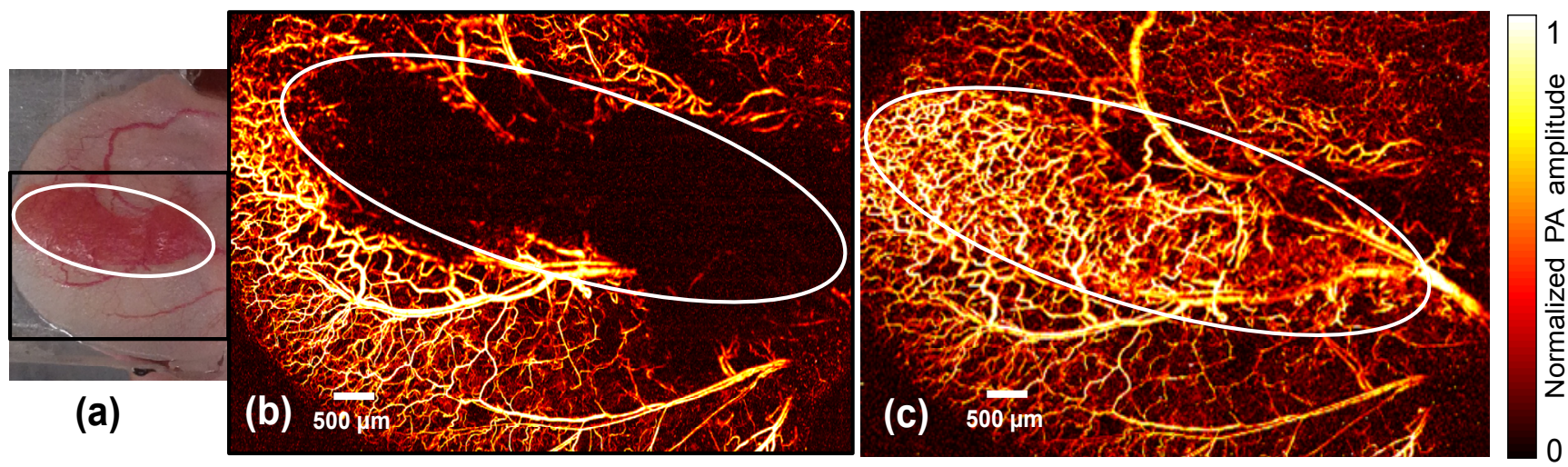

Figure 3. Comparison of mode 2 scanning at one depth and mode 3 scanningof a mouse ear in vivo. The white circles outline the tumor region. (a) Photo of a mouse ear with a growing tumor. In vivo MAP images acquired with COMSOR-PAM in (b) mode 2 and (c) mode 3. 

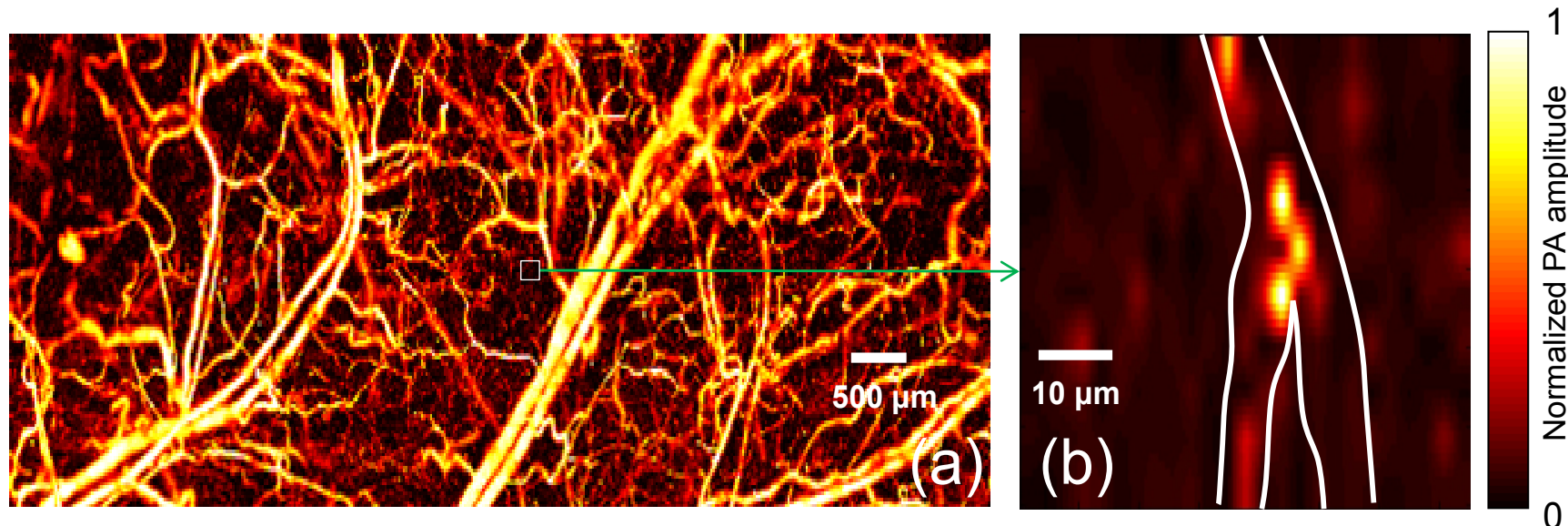

Figure 4. In vivo Real-time imaging of single red blood cells (RBCs) in a mouse ear. (a) MAP image acquired with COMS-OR-PAM in mode 2; (b) Tracking single RBCs in a selected capillary in vivo in mode 4.

High-speed label-free imaging of single red blood cells (RBCs) in vivo with millisecond-scale temporal resolution and micrometer-scale spatial resolution holds the key to uncovering the fundamental mechanisms of cellular metabolism [16]. To this end, a voice coil-based OR-PAM system with $100 \mathrm{~Hz}$ B-scan rate was invented [16], and a fiber bundle based OR-PAM system with $600 \mathrm{~Hz}$ B-scan rate was created [17].

Alternatively, a 2D galvanometer is employed in COMS-OR-PAM to achieve high-speed optical imaging within the acoustic focus (mode 4) at a laser repetition rate of $40 \mathrm{kHz}$. In vivo single cell tracking is enabled by the resulting B-scan imaging over a range of $50 \mu \mathrm{m}$ with $20 \mathrm{~A}$-lines at a rate of $2 \mathrm{kHz}$ or volumetric (3D) imaging over a FOV of $50 \times 50 \mu \mathrm{m} 2$ with 400 A-lines at a rate of $50 \mathrm{~Hz}$. The optical scanning range was set to $50 \mu \mathrm{m}$, smaller than the acoustic focus, to maintain a high SNR. Fig. 4(a) shows part of the ear vascular anatomy of a living nude mouse (Hsd: Athymic NudeFoxn1nu, Harlan Co.), imaged in mode 2. With the site map, mode 4 is further used to monitor a small region of interest, and Fig. 4(b) shows a single red blood cell (RBC) imaged in vivo in mode 4 . Mode 4 has achieved a B-scan imaging speed 20 times higher than voice-coil PAM, at the expense of FOV, which however is often not a concern if the target is cellular or capillary dynamics.

To achieve an optimal tradeoff between imaging speed and FOV, we implemented synchronized 1D optical and 2D mechanical hybrid scanning (mode 5). The scanning mechanism of mode 5 is illustrated in Fig. 5(a). In mode 5, the laser was working at a $40 \mathrm{kHz}$ repetition rate. The Y-axis galvanometer repeatedly scanned with a line-scan interval of $50 \mu \mathrm{m}$ and a line-scan rate of $2 \mathrm{kHz}$. The $\mathrm{X}$-axis motor was synchronized with the $\mathrm{Y}$-axis galvanometer and moved one-step forward after the $\mathrm{Y}$-axis galvanometer finished one line scan. After the X-axis motor finished one B-scan, the Y-axis motor moved in a large step size of $50 \mu \mathrm{m}$ (in comparison with a step size of 2.5-5.0 $\mu \mathrm{m}$ in mode 2). Fig. 5(b) shows the image of a mouse ear with a $10 \times 8 \mathrm{~mm}^{2}$ FOV acquired within 150 seconds in mode 5 . With the much enlarged scanning step of the Y-axis motor, the imaging speed of mode 5 is 20 times faster than that of the second-generation OR-PAM [14].

In summary, COMS-OR-PAM provides five scanning modes with high lateral resolution, fast imaging speed, and a wide FOV. Its capability of tracking in vivo cell activities in real time and imaging uneven surfaces will be invaluable for fast and quantitative microscopic studies of tumors and the brain as long as the region of interest is optically accessible. 

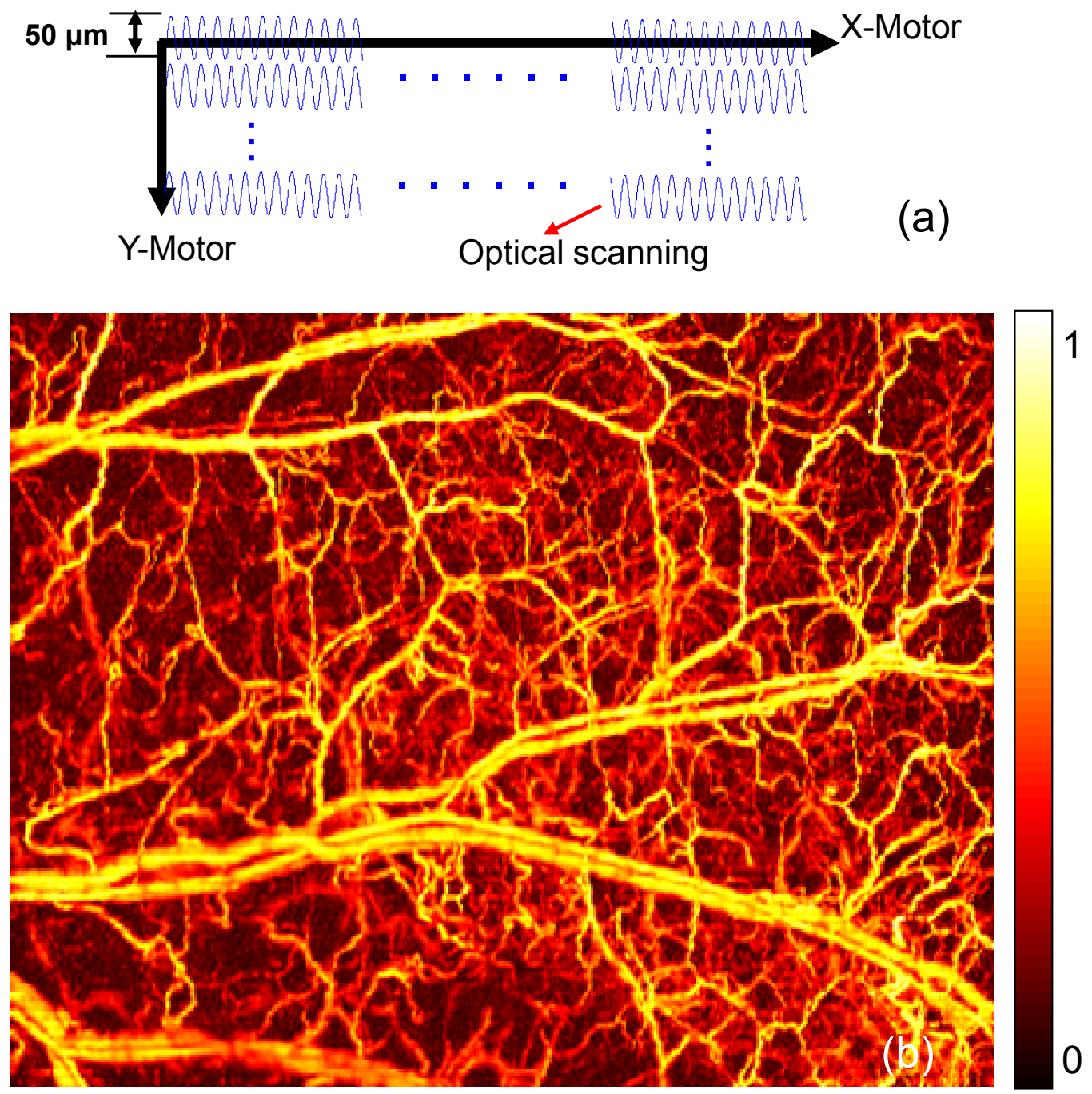

Figure 5. Mouse ear imaging in vivo with COMS-OR-PAM in mode 5. (a) Mechanism of the mode 5 scanning, (b) MAP image of the mouse ear vasculature acquired in mode 5.

\section{ACKNOWLEDGEMENTS}

The authors appreciate Prof. James Ballard's close reading of the manuscript, and thank Jinyang Liang, Chiye Li, and Yong Zhou for helpful discussions and experimental assistance. This work was sponsored in part by National Institutes of Health (NIH) grants DP1 EB016986 (NIH Director's Pioneer Award), R01 CA159959, and R01 CA134539. L.V. Wang has a financial interest in Microphotoacoustics Inc., and Endra Inc., which, however, did not support this work. K. I. Maslov has a financial interest in Microphotoacoustics Inc.

\section{REFERENCES}

[1] K. Maslov, H. F. Zhang, S. Hu, and L. V. Wang, "Optical-resolution photoacoustic microscopy for in vivo imaging of single capillaries," Optics Letters 33, 929-931 (2008).

[2] L. V. Wang, and S. Hu, "Photoacoustic tomography: in vivo imaging from organelles to organs," Science 335, 1458$1462(2012)$. 
[3] Y. Jiang, A. Forbrich, T. Harrison, and R. J. Zemp, "Blood oxygen flux estimation with a combined photoacoustic and high-frequency ultrasound microscopy system: a phantom study," Journal of Biomedical Optics 17, 036012 (2012).

[4] A. Ray, J. R. Rajian, Y. E. Lee, X. Wang, and R. Kopelman, "Lifetime-based photoacoustic oxygen sensing in vivo," Journal of Biomedical Optics 17, 057004 (2012).

[5] R. L. Shelton, and B. E. Applegate, "Ultrahigh resolution photoacoustic microscopy via transient absorption," Biomed. Opt. Express 1, 676-686 (2010).

[6] R. L. Shelton, Mattison, S. P. and Applegate, B. E., "Volumetric imaging of erythrocytes using label-free multiphoton photoacoustic microscopy," Journal of Biophotonics, 7 (2013).

[7] R. J. Paproski, A. E. Forbrich, K. Wachowicz, M. M. Hitt, and R. J. Zemp, "Tyrosinase as a dual reporter gene for both photoacoustic and magnetic resonance imaging," Biomed. Opt. Express 2, 771-780 (2011).

[8] G. Suffredini, J. E. East, and L. M. Levy, "New Applications of Nanotechnology for Neuroimaging," American Journal of Neuroradiology (2013).

[9] S. Hu, B. Rao, K. Maslov, and L. V. Wang, "Label-free photoacoustic ophthalmic angiography," Optics Letters 35, $1-3(2010)$.

[10] S. L. Jiao, M. S. Jiang, J. M. Hu, A. Fawzi, Q. F. Zhou, K. K. Shung, C. A. Puliafito, and H. F. Zhang, "Photoacoustic ophthalmoscopy for in vivo retinal imaging," Optics Express 18, 3967-3972 (2010).

[11]T. Liu, H. Li, W. Song, S. L. Jiao, and H. F. Zhang, "Fundus Camera Guided Photoacoustic Ophthalmoscopy," Current Eye Research. 38, 1229-1234 (2013).

[12] W. Song, Q. Wei, S. Jiao, and H. F. Zhang, "Integrated photoacoustic ophthalmoscopy and spectral-domain optical coherence tomography," Journal of Visualized Experiments: JoVE, e4390 (2013).

[13] S. S. Oladipupo, S. Hu, A. C. Santeford, J. J. Yao, J. R. Kovalski, R. V. Shohet, K. Maslov, L. V. Wang, and J. M. Arbeit, "Conditional HIF-1 induction produces multistage neovascularization with stage-specific sensitivity to VEGFR inhibitors and myeloid cell independence," Blood 117, 4142-4153 (2011).

[14] Song Hu, Konstantin Maslov, and a. L. V. Wang*, "Second-generation optical-resolution photoacoustic microscopy with improved sensitivity and speed," Optics Letters 36, 3 (2011).

[15]H. F. Zhang, K. Maslov, M. L. Li, G. Stoica, and L. H. V. Wang, "In vivo volumetric imaging of subcutaneous microvasculature by photoacoustic microscopy," Optics Express 14, 9317-9323 (2006).

[16] L. D. Wang, K. Maslov, and L. H. V. Wang, "Single-cell label-free photoacoustic flowoxigraphy in vivo," Proceedings of the National Academy of Sciences of the United States of America 110, 5759-5764 (2013).

[17] P. Hajireza, W. Shi, and R. J. Zemp, "Label-free in vivo fiber-based optical-resolution photoacoustic microscopy," Optics Letters 36, 4107-4109 (2011). 